\title{
Aspect Ratio Effect of Vertical Lid Driven Chamber Having a Centered Conducting Solid on Mixed Magnetoconvection
}

\author{
R. Nasrin* \\ Department of Mathematics, Bangladesh University of Engineering and Technology, Dhaka-1000, \\ Bangladesh
}

Received 12 April 2011, accepted in final revised form 26 June 2011

\begin{abstract}
The aspect ratio effect of vertical lid driven rectangular chamber on mixed magnetoconvective flow and heat transfer characteristics has been numerically studied. A heat conducting square solid body is centered of the chamber. The lower and upper surfaces of this chamber are adiabatic. The left vertical wall is mechanically lid driven having constant temperature $T_{i}$ and velocity $v_{0}$ while other side is sinusoidal wavy pattern and maintains higher temperature $T_{h}$ than the lid. Using Galerkin weighted residual finite element technique the governing two-dimensional flow equations have been solved. The investigations are conducted for different values of Richardson number (Ri) and aspect ratio $(A R)$ of chamber. Various characteristics such as streamlines, isotherms and heat transfer rate in terms of the mean Nusselt number $(\mathrm{Nu})$, the average temperature $\left(\theta_{a v}\right)$ of the fluid and temperature of solid center $\left(\theta_{c}\right)$ are presented. Maximum rate of heat transfer is observed for the lowest aspect ratio $A R$ owing to the shortest distance between the hot and cold surfaces.
\end{abstract}

Keywords: Mixed magnetoconvection; Wavy surface; Aspect ratio; Heat conducting solid; Lid driven chamber; Finite element simulation.

(C) 2011 JSR Publications. ISSN: 2070-0237 (Print); 2070-0245 (Online). All rights reserved.

doi:10.3329/jsr.v3i37433

J. Sci. Res. 3 (3), 501-513 (2011)

\section{Introduction}

The influence of the magnetic field on the convective heat transfer and the mixed convection flow of the fluid are of paramount importance in engineering. Joule heating in electronics and in physics refers to the increase in temperature of a conductor as a result of resistance to an electrical current flowing through it. For different aspect ratio of 2D or 3D geometry, there is a correlation with Nusselt number. The practical aim is usually to relate the heat transfer across the geometry to the temperature difference between the walls. A combined free and forced convection flow of an electrically conducting fluid in a cavity or in a channel in the presence of magnetic field is of special technical

\footnotetext{
*E-mail: rehena@math.buet.ac.bd
} 
significance because of its frequent occurrence in many industrial applications such as geothermal reservoirs, cooling of nuclear reactors, thermal insulations and petroleum reservoirs. These types of problems also arise in electronic packages, micro electronic devices during their operations.

Papanicolaou and Jaluria [1] carried out a numerical study to investigate the combined forced and natural convective cooling of heat dissipating electronic components, located in rectangular enclosure and cooled by an external flow of air. At the same year, House et al. [2] considered natural convection in a vertical square cavity with heat conducting body, placed on center in order to understand the effect of the heat conducting body on the heat transfer process in the cavity. They found that the heat transfer across the enclosure enhanced by a body with thermal conductivity ratio less than unity. Garandet et al. [3] studied natural convection heat transfer in a rectangular enclosure with a transverse magnetic field. Rudraiah et al. [4] investigated the effect of surface tension on buoyancy driven flow of an electrically conducting fluid in a rectangular cavity in the presence of a vertical transverse magnetic field to see how this force damped hydrodynamic movements. Calmidi and Mahajan [5] studied mixed convection in a partially divided rectangular enclosure over a wide range of Reynolds and Grashof numbers. Their findings were that the average Nusselt number and the dimensionless surface temperature depended on the location and height of the divider.

Combined free and forced convection in a square enclosure with heat conducting body and a finite-size heat source was simulated numerically by Hsu and How [6]. They concluded that both the heat transfer coefficient and the dimensionless temperature in the body center strongly depended on the configurations of the system. At the same time, Omri and Nasrallah [7] studied mixed convection in an air-cooled cavity with differentially heated vertical isothermal sidewalls having inlet and exit ports by a control volume finite element method. Results showed two different placement configurations of the inlet and exit ports on the sidewalls. The problem of unsteady laminar combined forced and free convection flow and heat transfer of an electrically conducting and heat generating or absorbing fluid in a vertical lid-driven cavity in the presence of magnetic field was formulated by Chamkha [8].

Shokouhmand and Sayehvand [9] carried out the numerical study of flow and heat transfer in a square driven cavity. They found that at the higher values of Reynolds number, an inviscid core region developed, but secondary eddies were present in the bottom corners of the square at all Reynolds numbers. Bhoite et al. [10] conducted numerically the problem of mixed convection flow and heat transfer in a shallow enclosure with a series of block-like heat generating component for a range of Reynolds and Grashof numbers and block-to-fluid thermal conductivity ratios. The authors declared that higher Reynolds number created a recirculation region of increasing strength at the core region. Al-Amiri et al. [11] performed mixed convection heat transfer in lid-driven cavity with a sinusoidal wavy bottom surface, where the corrugated liddriven cavity could be considered as an effective heat transfer mechanism at larger wavy surface amplitudes and low Richardson number. 
Recently, Oztop et al. [12] analyzed fluid flow due to combined convection in liddriven enclosure having a circular body. Mixed convection in an obstructed open-ended cavity with heated horizontal walls was analyzed by Shi and Vafai [13]. They declared that as the aspect ratio increased the thickness of the thermal boundary layer increased, resulted in a decrease in the heat transfer rate though the horizontal walls. Very recently, mixed convection in a square cavity of sinusoidal boundary conditions at the sidewalls in the presence of magnetic field was investigated numerically by Sivasankaran et al. [14]. They concluded that the heat transfer rate increased with the phase deviation up to $\phi=\pi / 2$ and then it decreased for further increase in the phase deviation.

In the light of the above literature, it has been pointed out that there are no significant information about aspect ratio effect of a wavy chamber having heat conducting solid on the mixed convection processes. The present study addresses this issue. Numerical solutions are obtained over a wide range of $R i$ and $A R$. Results are presented graphically in terms of streamlines and isothermal lines.

\section{Problem Formulation}

The geometry of the problem with necessary boundary conditions herein investigated is depicted in Fig. 1. The base and top part of the cavity are well insulated. It is assumed that the left lid is at a uniform velocity $v_{0}$ and at the temperature $T_{i}$. As well the right vertical corrugated wall is more heated than the left lid with the temperature $T_{h}$. A square heat conducting solid is centered of the chamber. The functioning fluid is assumed as air $(P r=0.73)$. A magnetic field of strength $B_{0}$ is applied in the transverse direction to the side walls of the cavity. In addition Joule heating effect is considered.

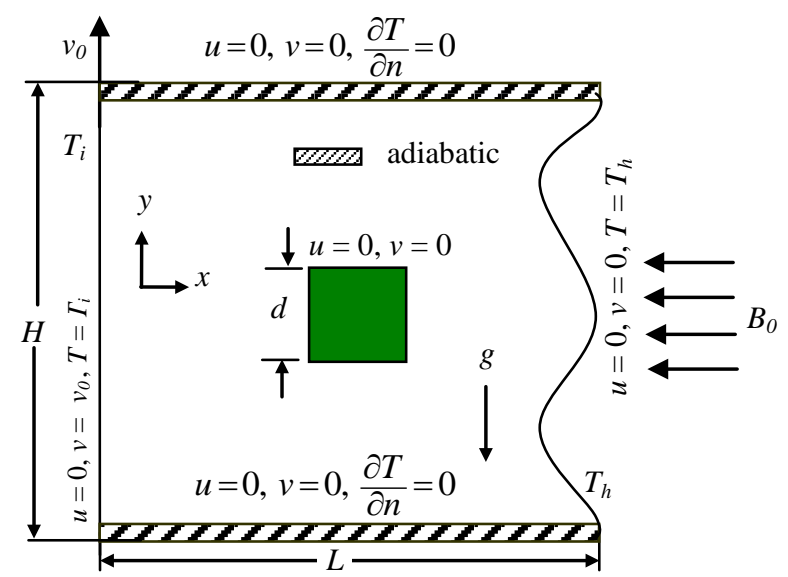

Fig. 1. Schematic diagram of chamber and boundary conditions.

\section{Mathematical Formulation}

A two-dimensional, steady, laminar, incompressible, mixed convection flow is considered within the chamber and the fluid properties are assumed to be constant. The 
radiation effect is taken as negligible. The dimensional equations describing the flow under Boussinesq approximation used $[10,11]$ are as follows:

$$
\begin{aligned}
& \frac{\partial u}{\partial x}+\frac{\partial v}{\partial y}=0 \\
& u \frac{\partial u}{\partial x}+v \frac{\partial u}{\partial y}=-\frac{1}{\rho} \frac{\partial p}{\partial x}+v\left(\frac{\partial^{2} u}{\partial x^{2}}+\frac{\partial^{2} u}{\partial y^{2}}\right) \\
& u \frac{\partial v}{\partial x}+v \frac{\partial v}{\partial y}=-\frac{1}{\rho} \frac{\partial p}{\partial y}+v\left(\frac{\partial^{2} v}{\partial x^{2}}+\frac{\partial^{2} v}{\partial y^{2}}\right)+g \beta\left(T-T_{i}\right)-\frac{\sigma B_{0}{ }^{2} v}{\rho} \\
& u \frac{\partial T}{\partial x}+v \frac{\partial T}{\partial y}=\frac{k}{\rho c_{p}}\left(\frac{\partial^{2} T}{\partial x^{2}}+\frac{\partial^{2} T}{\partial y^{2}}\right)+\frac{\sigma B_{0}{ }^{2} v^{2}}{\rho c_{p}} \\
& \frac{k_{s}}{\rho c_{p}}\left(\frac{\partial^{2} T_{s}}{\partial x^{2}}+\frac{\partial^{2} T_{s}}{\partial y^{2}}\right)=0
\end{aligned}
$$

For the present phenomena the boundary conditions are specified as follows:

at the vertical lid: $u=0, v=v_{0}, T=T_{i}$

at the horizontal walls: $u=0, v=0, \frac{\partial T}{\partial n}=0$

at the wavy surface: $u=0, v=0, T=T_{h}$

at all cylinder boundaries: $u=0, v=0$

at the solid-fluid interface: $\left(\frac{\partial T}{\partial n}\right)_{\text {fluid }}=\frac{k_{s}}{k}\left(\frac{\partial T_{s}}{\partial n}\right)_{\text {solid }}$

The rate of heat transfer is computed at the wavy wall and is expressed in terms of the local Nusselt number $\overline{N u}$ as $\overline{N u}=\frac{h L}{k}=-\frac{\partial T}{\partial n} L$, where $h$ and $n$ are the local convective heat transfer coefficient and dimensional distances either along $x$ or $y$ direction acting normal to the surface respectively. The above equations are non-dimensionalized by using the following dimensionless quantities

$$
X=\frac{x}{L}, Y=\frac{y}{L}, U=\frac{u}{v_{0}}, V=\frac{v}{v_{0}}, D=\frac{d}{L}, P=\frac{p}{\rho v_{0}^{2}}, \theta=\frac{\left(T-T_{i}\right)}{\left(T_{h}-T_{i}\right)}, \theta_{S}=\frac{\left(T_{S}-T_{i}\right)}{\left(T_{h}-T_{i}\right)}
$$

Then the non-dimensional governing equations are

$$
\begin{aligned}
& \frac{\partial U}{\partial X}+\frac{\partial V}{\partial Y}=0 \\
& U \frac{\partial U}{\partial X}+V \frac{\partial U}{\partial Y}=-\frac{\partial P}{\partial X}+\frac{1}{R e}\left(\frac{\partial^{2} U}{\partial X^{2}}+\frac{\partial^{2} U}{\partial Y^{2}}\right)
\end{aligned}
$$




$$
\begin{aligned}
& U \frac{\partial V}{\partial X}+V \frac{\partial V}{\partial Y}=-\frac{\partial P}{\partial Y}+\frac{1}{\operatorname{Re}}\left(\frac{\partial^{2} V}{\partial X^{2}}+\frac{\partial^{2} V}{\partial Y^{2}}\right)+\operatorname{Ri} \theta-\frac{H a^{2}}{R e} V \\
& U \frac{\partial \theta}{\partial X}+V \frac{\partial \theta}{\partial Y}=\frac{1}{\operatorname{RePr}}\left(\frac{\partial^{2} \theta}{\partial X^{2}}+\frac{\partial^{2} \theta}{\partial Y^{2}}\right)+J V^{2} \\
& \frac{K}{\operatorname{RePr}}\left(\frac{\partial^{2} \theta_{S}}{\partial X^{2}}+\frac{\partial^{2} \theta_{s}}{\partial Y^{2}}\right)=0
\end{aligned}
$$

Here $\operatorname{Re}=\frac{v_{0} L}{v}, \operatorname{Pr}=\frac{v}{\alpha}, R i=\frac{g \beta\left(T_{h}-T_{i}\right) L}{v_{i}{ }^{2}}, K=k_{s} / k$ and $J=\frac{\sigma B_{0}^{2} \alpha}{\rho C_{p}\left(T_{h}-T_{i}\right)}$ are Reynolds number, Prandtl number, Richardson number, solid-fluid thermal conductivity ratio and Joule heating parameter respectively. $\mathrm{Ha}$ is Hartmann number which is defined as $H a^{2}=\frac{\sigma B_{0}^{2} L^{2}}{\mu}$. The boundary conditions for the current problem are specified as follows: at the left lid: $U=0, V=1, \theta=0$

at the horizontal surfaces: $U=0, V=0, \frac{\partial \theta}{\partial N}=0$

at the wavy wall: $U=V=0, \theta=1$

at the solid boundary: $U=V=0$

at the fluid-solid interface: $\left(\frac{\partial \theta}{\partial N}\right)_{\text {fluid }}=K\left(\frac{\partial \theta_{S}}{\partial N}\right)_{\text {solid }}$.

The nature of the vertical wavy surface profile is assumed the pattern of $X-1=-A[1-\cos (2 \lambda \pi Y)]$, where $A$ is the dimensionless amplitude of the corrugated surface and $\lambda$ is the number of undulations. The local Nusselt number at the heated corrugated surface is $\overline{N u}=-\frac{\partial \theta}{\partial N} L$. The normal temperature gradient can be written as $\frac{\partial \theta}{\partial N}=\frac{1}{L} \sqrt{\left(\frac{\partial \theta}{\partial X}\right)^{2}+\left(\frac{\partial \theta}{\partial Y}\right)^{2}}$. The average Nusselt number $(\mathrm{Nu})$ is obtained by integrating the local Nusselt number along the vertical wavy surface and is defined by $N u=\frac{1}{S} \int_{0}^{S} \overline{N u} d N$, where $S$ is the total dimensionless chord length of the wavy surface.

The mean temperature of the fluid is $\theta_{a v}=\int \theta d \bar{V} / \bar{V}$, where the volume of the chamber is $\bar{V}$. As well aspect ratio of the vertical lid driven chamber is $A R=L / H$

\section{Finite Element Simulation}

The momentum and energy balance equations have been solved numerically based on the Galerkin weighted residual finite element technique of Dechaumphai [16]. The velocity components $U$ and $V$, the temperature $\theta$ and the pressure $P$ are the basic unknowns in the 
above differential equations. The six node triangular element is used in this work for the development of the finite element equations. All six nodes are associated with velocities as well as temperature. Only the three corner nodes are associated with pressure. The velocity components, the temperature and linear interpolation for the pressure distribution according to their highest derivative orders in Eqs. (6) - (10) are as follows:

$U(X, Y)=N_{\beta} U_{\beta}, V(X, Y)=N_{\beta} V_{\beta}, \theta(X, Y)=N_{\beta} \theta_{\beta}, \theta_{s}(X, Y)=N_{\beta} \theta_{s_{\beta}}, P(X, Y)=H_{\lambda} P_{\lambda}$, where $\beta=1,2, \ldots \ldots, 6$ and $\lambda=1,2,3$.

By substituting the element velocity components, temperature and pressure distributions in Eqs. (6) - (10), the modified finite element equations are

$$
\begin{aligned}
& K_{\alpha \beta^{x}} U_{\beta}+K_{\alpha \beta^{y}} V_{\beta}=0 \\
& K_{\alpha \beta \gamma^{X}} U_{\beta} U_{\gamma}+K_{\alpha \beta \gamma}{ }^{y} V_{\gamma} U_{\gamma}+M_{\alpha \mu^{X}} P_{\mu}+\frac{1}{R e}\left(S_{\alpha \beta^{X X}}+S_{\alpha \beta} y y\right) U_{\beta}=Q_{\alpha} u \\
& K_{\alpha \beta \gamma^{x}} U_{\beta} V_{\gamma}+K_{\alpha \beta \gamma} y V_{\gamma} V_{\gamma}+M_{\alpha \mu}{ }^{y} P_{\mu}+\frac{1}{R e}\left(S_{\alpha \beta^{x x}}+S_{\alpha \beta}^{y y}\right) V_{\beta}- \\
& R i K_{\alpha \beta} \theta_{\beta}+\frac{H a^{2}}{R e} K_{\alpha \beta} V_{\beta}=Q_{\alpha} v \\
& K_{\alpha \beta \gamma}{ }^{x} U_{\beta} \theta_{\gamma}+K_{\alpha \beta \gamma} y V_{\beta} \theta_{\gamma}+\frac{1}{\operatorname{RePr} P}\left(S_{\alpha \beta^{x X}}+S_{\alpha \beta} y y\right) \theta_{\beta}-J K_{\alpha \beta} V_{\beta}^{2}=Q_{\alpha} \theta \\
& \frac{K}{\operatorname{RePr} P}\left(S_{\alpha \beta^{X X}}+S_{\alpha \beta} y y\right) \theta_{\beta}=Q_{\alpha} \theta_{S}
\end{aligned}
$$

where the coefficients in element matrices of the integrals over the element area and along the element edges $S_{0}$ and $S_{w}$ are as

$$
\begin{aligned}
& K_{\alpha \beta^{x}}=\int_{A} N_{\alpha} N_{\beta, x} d A, K_{\alpha \beta^{y}}=\int_{A} N_{\alpha} N_{\beta, y} d A, K_{\alpha \beta \gamma^{x}}=\int_{A} N_{\alpha} N_{\beta} N_{\gamma, x} d A, \\
& K_{\alpha \beta \gamma^{y}}=\int_{A} N_{\alpha} N_{\beta} N_{\gamma, y} d A, K_{\alpha \beta}=\int_{A} N_{\alpha} N_{\beta} d A, S_{\alpha \beta^{x x}}=\int_{A} N_{\alpha, x} N_{\beta, x} d A, \\
& S_{\alpha \beta^{y y}}=\int_{A} N_{\alpha, y} N_{\beta, y} d A, M_{\alpha \mu^{x}}=\int_{A} H_{\alpha} H_{\mu, x} d A, M_{\alpha \mu^{y}}=\int_{A} H_{\alpha} H_{\mu, y} d A, \\
& Q_{\alpha^{u}}=\int_{S_{0}} N_{\alpha} S_{x} d S_{0}, Q_{\alpha}{ }^{v}=\int_{S_{0}} N_{\alpha} S_{y} d S_{0}, Q_{\alpha} \theta=\int_{S_{w}} N_{\alpha} q_{1_{w}} d S_{w}, \\
& Q_{\alpha^{\theta}{ }_{s}}=\int_{S_{w}} N_{\alpha} q_{2_{w}} d S_{w}
\end{aligned}
$$

Using Newton-Raphson method of Reddy [17], the set of non-linear algebraic Eqs. (11) - (15) are transferred into linear algebraic equations. Finally, these linear equations are solved by applying Triangular Factorization method and reduced integration technique of Zeinkiewicz et al. [18].

\section{Grid Refinement Test}

A grid independence test is reported with $H a=12, R e=100, K=7, A=0.05, D=0.2, \lambda$ $=2, R i=0.1, J=2$ and $\operatorname{Pr}=0.73$ in order to decide the suitable grid size for this study. 
The extreme values of the mean Nusselt number $(\mathrm{Nu})$ that relates to the heat transfer rate of the heated surface and average temperature $\left(\theta_{a v}\right)$ of the fluid in the chamber are used as sensitivity measures of the correctness of the solution. The addiction of the quantities $\mathrm{Nu}$ and $\theta_{a v}$ on the grid size and the computational time are shown in Table 1. Considering both the accuracy of numerical values and computational time, the current formulation is performed with 31514 nodes and 3636 elements grid system.

Table 1. Grid sensitivity check at $H a=12, \operatorname{Pr}=0.73, R i=0.1, R e=100, A=0.05$, $K=7, \lambda=2, J=2$ and $D=0.2$.

\begin{tabular}{cccccc}
\hline $\begin{array}{c}\text { Nodes } \\
\text { (elements) }\end{array}$ & $\begin{array}{c}3918 \\
(436)\end{array}$ & $\begin{array}{c}9373 \\
(1064)\end{array}$ & $\begin{array}{c}15465 \\
(1768)\end{array}$ & $\begin{array}{c}31514 \\
(3636)\end{array}$ & $\begin{array}{c}49592 \\
(5748)\end{array}$ \\
\hline $\mathrm{Nu}$ & 1.597893 & 1.602778 & 1.618247 & 1.622647 & 1.622647 \\
$\theta_{a v}$ & 0.336241 & 0.325121 & 0.310542 & 0.306112 & 0.306112 \\
Time (s) & 246.269 & 308.603 & 398.167 & 445.321 & 550.379 \\
\hline
\end{tabular}

\section{Mesh Generation}

In finite element method, the mesh generation is the technique to subdivide a domain into a set of sub-domains, called finite elements, control volume etc. The discrete locations are defined by the numerical grid, at which the variables are to be calculated. The computational domains with irregular geometries by a collection of finite elements make the method a valuable practical tool for the solution of boundary value problems arising in various fields of engineering. Fig. 2 displays the finite element mesh of the present physical domain.

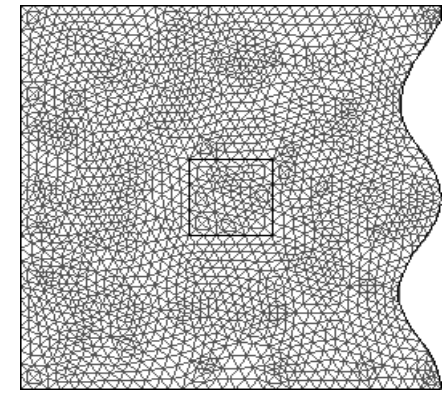

Fig. 2. Mesh structure for wavy chamber.

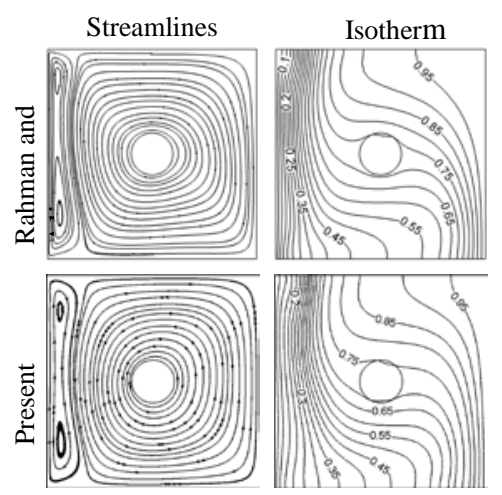

Fig. 3. Comparison between present work and Rahman and Alim [15].

\section{Code Validation}

The model validation is a necessary part of a mathematical investigation. The outcome of the present numerical code is benchmarked against the numerical result of Rahman and 
Alim [15] which was reported for MHD mixed convection flow in a vertical lid-driven square enclosure including a heat conducting horizontal circular cylinder with Joule heating. The comparison is conducted while employing the dimensionless parameters $R e$ $=100, R i=5.0, K=5.0, H a=10.0, J=1.0, D=0.2$ and $P r=0.71$. Present result for both the streamlines and isotherms is shown in Fig. 3, which is an outstanding agreement with those of [15]. This justification boosts the assurance in this numerical code to carry on with the above stated objective of the existing investigation.

\section{Results and Discussion}

A numerical computation has been carried out through finite element simulation to analyze the aspect ratio effect of horizontal wavy chamber having a centered heat conducting solid on mixed magnetoconvection flow. Effects of the controlling parameters such as $R i$ and $A R$ on heat transfer and fluid flow inside the chamber have been studied. In order to investigate the convective heat transfer behavior, computation is done for chamber at four aspect ratios of $0.5,1,1.5$ and 2.0 in the three convection regions $(R i=$ $0.1,1$ and 10). Joule heating parameter $J(=2)$, Hartmann number $\mathrm{Ha}(=12)$, Reynolds number $R e(=100)$ and thermal conductivity ratio $K(=7)$ are kept as invariable.

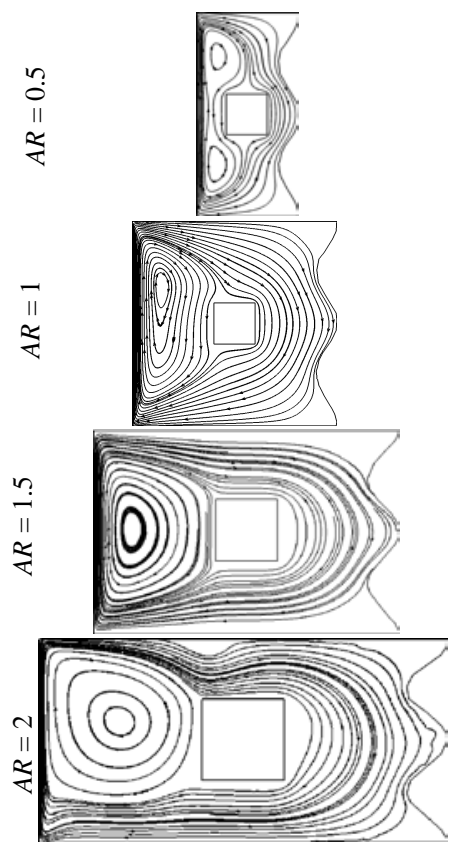

(a)

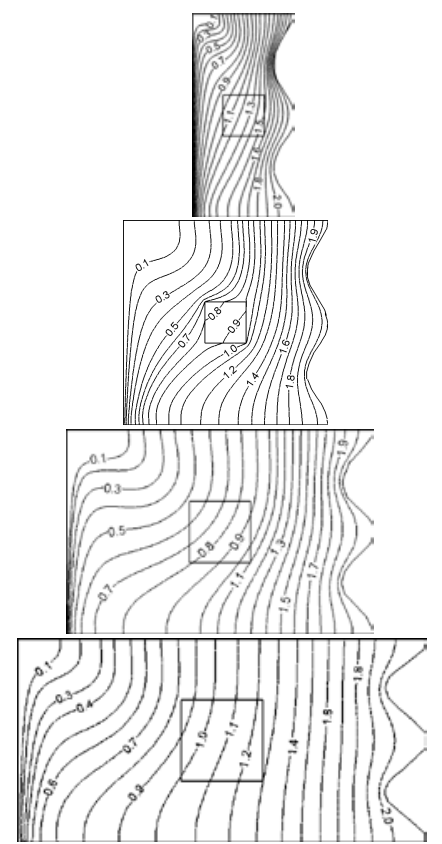

(b)

Fig. 4. (a) Streamlines and (b) Isotherms for various $A R$ at $R i=0.1$. 
The influence of the cavity aspect ratio on the velocity and thermal field in the chamber is revealed in Figs. 4(a) and (b) at forced convective regime $(R i=0.1)$. Due to the movement of the left lid, a bi-cellular primary vortex is produced at $A R=0.5$. With rising $A R$ it is noted from the Fig. 4(a) that the eddy becomes uni-cellular and large in size. The isothermal lines nearly follow the geometry of the right vertical corrugated surface for each aspect ratio. They start to turn back towards the hot wavy wall at the left top corner of the chamber. This is because the dominating effect of conduction and forced convection heat transfer.

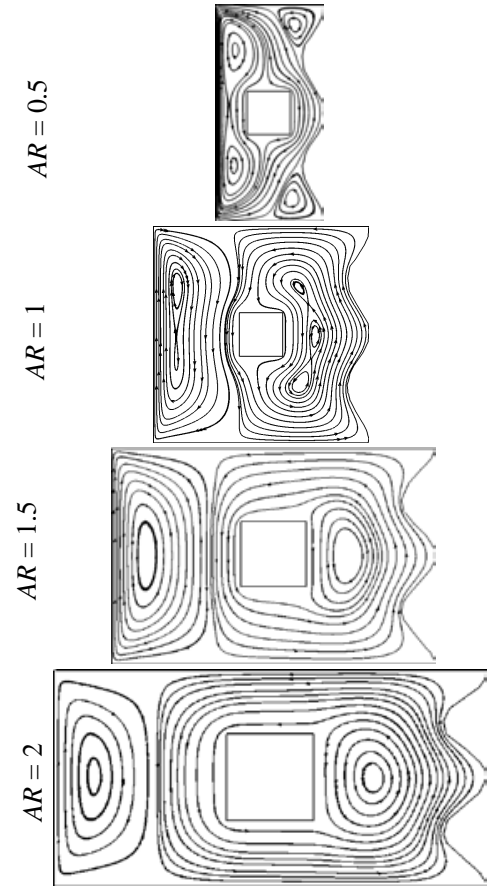

(a)

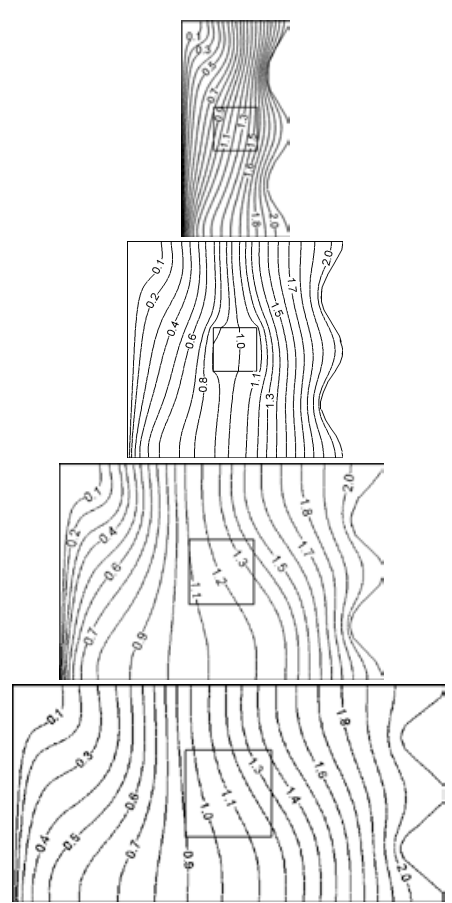

(b)

Fig. 5. Effect of $A R$ on (a) Streamlines and (b) Isotherms at $R i=1$.

Figs. 5(a) and (b) depict the effect of $A R$ at $R i=1$ on streamlines and isotherms. Due to the natural convection effect, the primary vortex remains unchanged and two secondary vortices are developed at the top and bottom corner near the right wall at $A R=$ 0.5. The size of the secondary eddy increases speedily as a result the size of the main vortex devalues quickly with growing $A R$. No major dissimilarity is found at lower $A R$ in a comparison of thermal current activities for previous case $(R i=0.1)$, but for higher $A R$ the isothermal lines start to turn back towards the cold wall in the upper part of the cavity. 
At $R i=10$ (free convection dominating region) and different values of $A R$, the velocity and temperature fields are plotted in Fig. 6(a) and (b), respectively. It is seen that the minor eddy spreads and thereby squeezes the primary vortex. It indicates a symbol of primacy of free convection in the chamber. The corresponding isothermal lines are almost parallel to the left vertical lid and a concentrated thermal boundary layer near the cold wall is developed at the lowest $A R$. But, for higher values of $A R(1.0,1.5$ and 2.0), this boundary layer becomes less compressed. Due to the strong influence of the convective current, a significant convective distortion in the temperature field occurs.

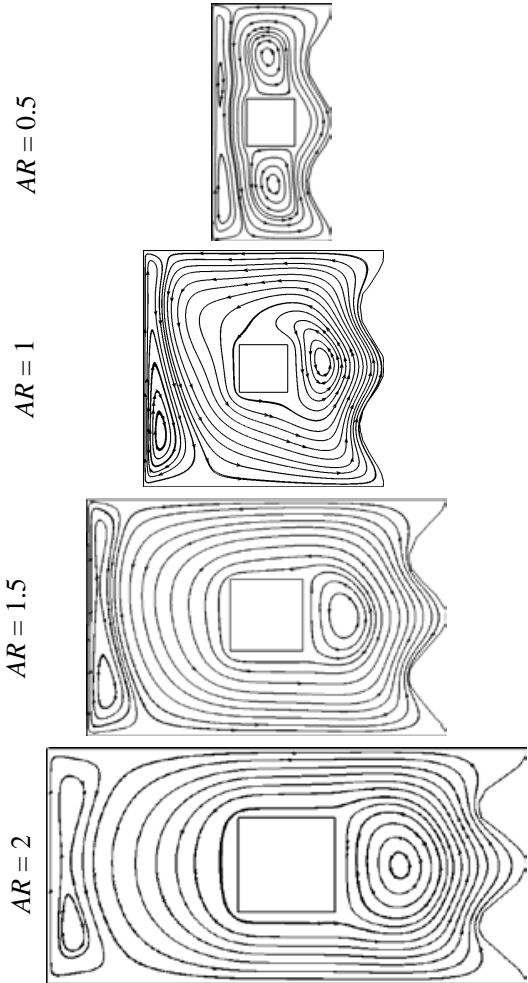

(a)

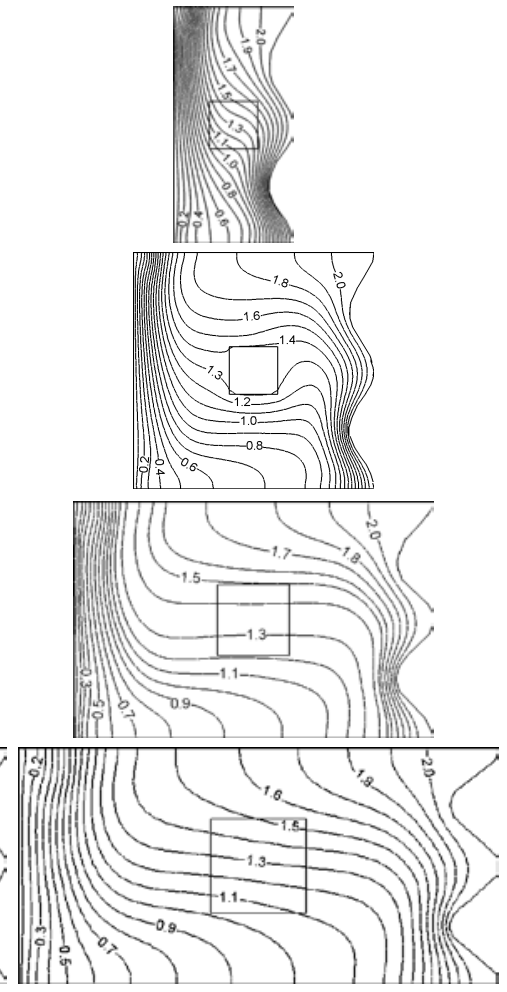

(b)

Fig. 6. (a) Streamlines and (b) Isotherms for different values of $A R$ at $R i=10$.

Figs. 7 (a)-(c) express the influence of $A R$ on the average Nusselt number $(\mathrm{Nu})$ at the heat source, average temperature $\left(\theta_{a v}\right)$ of the fluid in the chamber and the temperature $\left(\theta_{c}\right)$ at the solid center. They are displayed as a function of Richardson number. For higher $A R$, the $N u$-Ri profile shows two separate zones depending on Ri. In the forced convection dominated region $(R i<1), N u$ goes down sharply and it goes up gradually in the free convection dominated regime $(R i>1)$. On the other hand, for $A R=0.5$, it decreases monotonically with increasing $R i$. However, maximum rate of heat transfer is 
found for the lowest $A R(=0.5)$ at each $R i$. This happens due to the minimum distance between the hot and cold wall. For mounting $R i$, mean temperature of the fluid in the chamber and the temperature at the solid center rise sharply for the higher values of $A R$ (1.0, 1.5 and 2.0) and gradually at $A R=0.5$. In addition, they are lower for the higher values of $A R$ in the forced convection dominated region. They are least for the lowest $A R$ in the natural convection dominated regime.

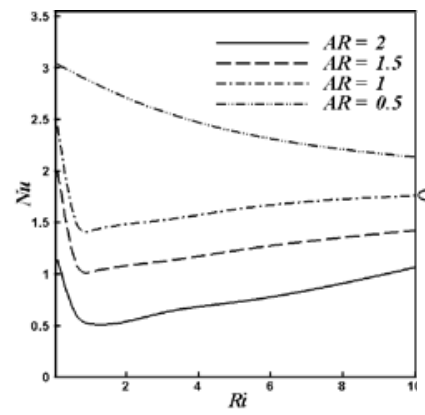

(a)

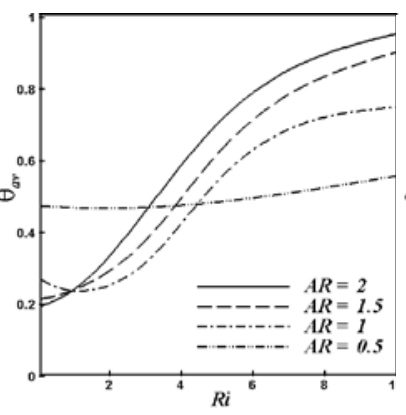

(b)

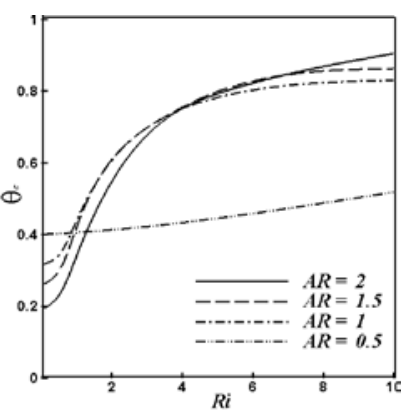

(c)

Fig. 7. Effect of $A R$ on (a) $N u$, (b) $\theta_{a v}$ and (c) $\theta_{c}$, while $R e=100, H a=12, J=2$ and $K=7$.

\section{Conclusion}

A computational study is performed to analyze the aspect ratio effect of a vertical lid driven chamber in presence of magnetic field. The following conclusions may be drawn from the foregoing investigations:

a. Cavity aspect ratio has noteworthy effects on the velocity and temperature profiles.

Buoyancy-induced vortex in the streamlines and convective current in the isotherms increase with mounting aspect ratio of the cavity. In addition, noticeably different flow behaviors and heat transfer characteristics are observed among the three different flow regimes.

b. Average Nusselt number is always higher for lowest value of the cavity aspect ratio. Average temperature of the fluid in the cavity and temperature at the cylinder center are lower in the forced convection dominated region for the higher values of $A R$ and in the free convection dominated region for the lowest value of $A R$.

\section{Appendix: Nomenclature}

A amplitude of wavy surface

$A R \quad$ aspect ratio of the chamber

$B_{0} \quad$ magnetic induction $\left(\mathrm{Wbm}^{-2}\right)$

$C_{p} \quad$ specific heat at constant pressure $\left(\mathrm{Jkg}^{-1} \mathrm{~K}^{-1}\right)$

$d \quad$ diameter of obstacle (m)

D non dimensional diameter of obstacle

$g \quad$ gravitational acceleration $\left(\mathrm{ms}^{-2}\right)$

$h \quad$ convective heat transfer coefficient $\left(\mathrm{Wm}^{-2} \mathrm{~K}^{-1}\right)$ 


\section{Aspect Ratio Effect}

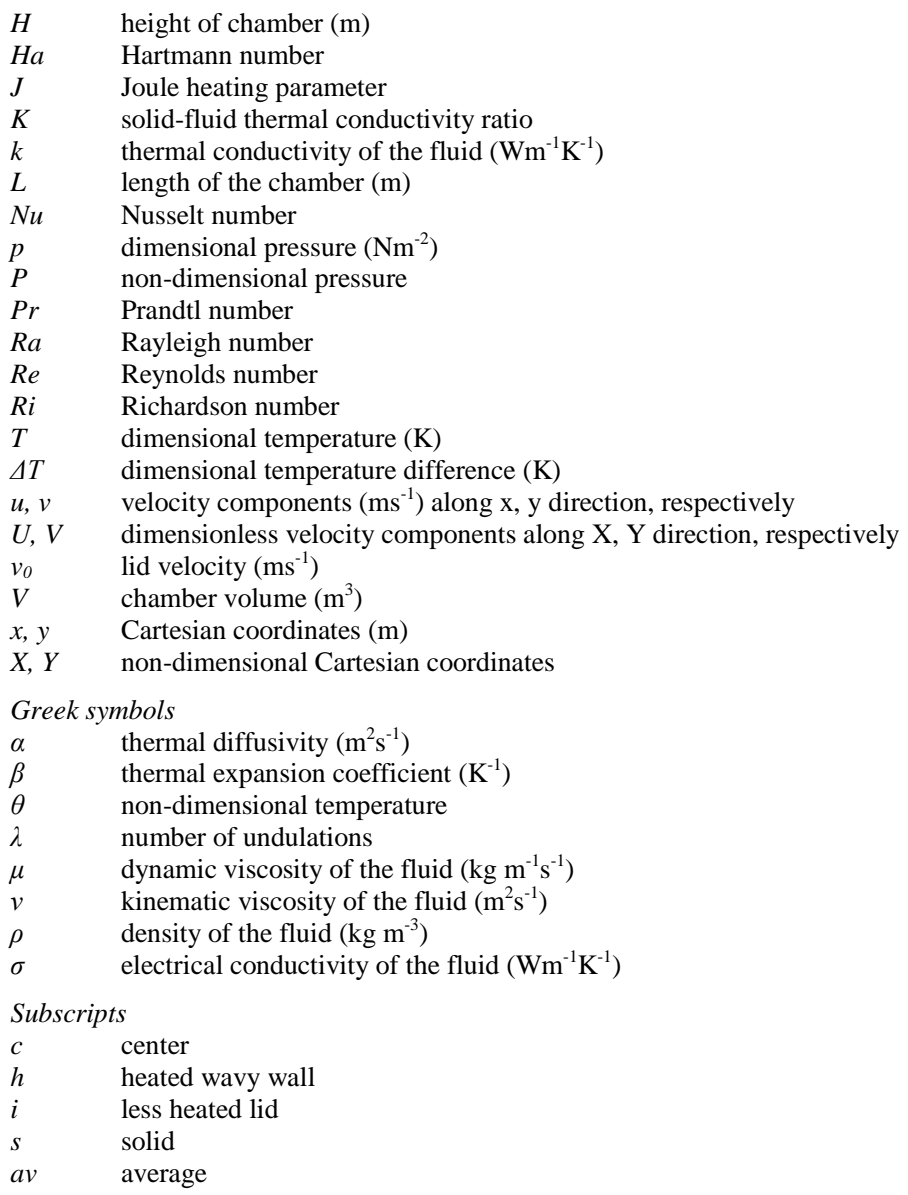

\section{References}

1. E. Papanicolaou, Y. Jaluria, Numerical Heat Transfer (Part A) 18, 427 (1990). http://dx.doi.org/10.1080/10407789008944802

2. J. M. House, C. Beckermann, T. F. Smith, Numerical Heat Transfer (Part A) 18, 213 (1990). http://dx.doi.org/10.1080/10407789008944791

3. J. P. Garandet, T. Alboussiere, R. Moreau, Int. J. of Heat and Mass Transfer 35, 741 (1992). http://dx.doi.org/10.1016/0017-9310(92)90242-K

4. N. Rudraiah, M. Venkatachalappa, C.K. Subbaraya, Int. J. of Non-linear Mechs. 30, 759 (1995). http://dx.doi.org/10.1016/0020-7462(95)00026-K

5. V. V. Calmidi, R. L. Mahajan, Int. J. of Heat and Fluid Flow 19, 358 (1998). http://dx.doi.org/10.1016/S0142-727X(98)00002-2

6. T. H. Hsu and S. P. How, Acta Mechanica 133, 87 (1999). http://dx.doi.org/10.1007/BF01179012

7. A. Omri, and S.B. Nasrallah, Numer. Heat Transfer (Part A) 36, 615 (1999). http://dx.doi.org/10.1080/104077899274606

8. A.J. Chamkha, Numer. Heat Transfer (Part A) 41, 529 (2002). http://dx.doi.org/10.1080/104077802753570356 
9. H. Shokouhmand and H. Sayehvand, Int. J. of Engg. (Transactions A): Basics 17, 301 (2004).

10. M. T. Bhoite, G. S. V. L Narasimham, and M. V. K Murthy, Int. J. of Thermal Sciences 44, 121 (2005). http://dx.doi.org/10.1016/j.ijthermalsci.2004.07.003

11. A. Al-Amiri, K. Khanafer, J. Bull, Ioan Pop, Int. J. of Heat and Mass Transfer 50, 1771 (2007). http://dx.doi.org/10.1016/j.ijheatmasstransfer.2006.10.008

12. H. F. Oztop, Z. Zhao, B. YU, Int. J. Heat Fluid Flow 30, 886 (2009). http://dx.doi.org/10.1016/j.ijheatfluidflow.2009.04.009

13. W. Shi, K. Vafai, Numer. Heat Transfer (Part A) 57, 709 (2010). http://dx.doi.org/10.1080/10407781003800714

14. S. Sivasankaran, A. Malleswaran, Jinho Lee, and P. Sundar, Int. J. of Heat and Mass Transfer 54, 512 (2011). http://dx.doi.org/10.1016/j.ijheatmasstransfer.2010.09.018

15. M. M. Rahman and M. A. Alim, Nonlinear Analysis: Modelling and Control 15, 199 (2010).

16. P. Dechaumphai, Finite Element Method in Engineering, 2nd Edition (Chulalongkorn University Press, Bangkok, 1999).

17. J. N. Reddy, An Introduction to Finite Element Analysis (McGraw-Hill, New York, 1993).

18. O.C. Zeinkiewicz, R. L. Taylor, J. M. Too, Int. J. for Num. Methods in Engg. 3, 275 (1971). 